\title{
SC-FDE for Offset Modulations: An Efficient Transmission Technique for Broadband Wireless Systems
}

\author{
Miguel Luzio, Student Member, IEEE, Rui Dinis, Member, IEEE, and Paulo Montezuma
}

\begin{abstract}
It is widely accepted that SC-FDE (Single-Carrier with Frequency-Domain Equalization) is an excellent candidate for broadband wireless systems, especially when an efficient power amplification is intended. If grossly nonlinear power amplifiers are employed, conventional QPSK (Quaternary Phase Shift Keying) or QAM (Quadrature Amplitude Modulation) modulations should be replaced by offset modulations such as OQPSK (Offset QPSK) and OQAM (Offset QAM). In fact, offset signals have much lower dynamic range than non-offset signals, with OQPSK signals being able to have an almost constant envelope.

This paper considers frequency-domain receiver design for OQPSK and OQAM schemes. It is shown that FDE (Frequency Domain Equalization) designed for non-offset modulations are not suitable for offset modulations due to the residual IQI (Inphase/Quadrature Interference), i.e. the interference between in-phase (I) and quadrature (Q) components at the sampling instants. Therefore, we propose several FDE designs where there is no IQI in the sampling instants as well as iterative FDE receivers with IQI cancellation. Our receivers have excellent performance, with the linear designs significantly outperforming linear FDE for non-offset modulations and the iterative designs with performance close to the MFB (Matched Filter Bound).
\end{abstract}

Index Terms-OQPSK modulations, SC-FDE, IQI, iterative receivers.

\section{INTRODUCTION}

$\mathbf{F}$ UTURE broadband wireless systems are expected to have high transmission rates while requiring good power and spectral efficiencies. To reduce the cost of mobile terminals, its complexity should be kept to a minimum. Moreover, an

Paper approved by G. M. Vitetta, the Editor for Equalization and Fading Channels of the IEEE Communications Society. Manuscript received February 4, 2011; revised June 30 and November 21, 2011.

This work was supported by the FCT/MEC (CTS multi-annual funding project PEst-OE/EEI/UI0066/2011, IT multi-annual funding project PEstOE/EEI/LA0008/2011, MPSat project PTDC/EEA-TEL/099074/2008, ADCOD project PTDC/EEA-TEL/099973/2008 and doctoral grant SFRH/BD/66172/2009).

M. Luzio is with the Dep. de Eng. Electrotécnica, Faculdade de Ciências e Tecnologia, FCT, Universidade Nova de Lisboa, 2829-516 Caparica, Portugal (e-mail: jmluzio@gmail.com). He is also with the Instituto de Telecomunicações, Portugal, and Uninova - CTS, Universidade Nova de Lisboa, Portugal.

R. Dinis is with IT, Instituto de Telecomunicações, Portugal. He is also with Dep. de Eng. Electrotécnica, Faculdade de Cincias e Tecnologia, FCT, Universidade Nova de Lisboa, 2829-516 Caparica, Portugal.

P. Montezuma is with Uninova - CTS, Universidade Nova de Lisboa, Portugal. He is also with with the Dep. de Eng. Electrotécnica, Faculdade de Ciências e Tecnologia, FCT, Universidade Nova de Lisboa, 2829-516 Caparica, Portugal.

Digital Object Identifier 10.1109//TCOMM.2012.051712.100072 efficient, low-cost power amplification is required, to increase the coverage and/or battery autonomy.

Due to high transmission rates, the multi-path propagation can lead to severe time-dispersion effects. For this reason, block transmission techniques combined with FDE (Frequency Domain Equalization) techniques such as OFDM (Orthogonal Frequency Division Multiplexing) [1] and SC-FDE (Single Carrier Frequency Domain Equalization) [2] should be employed. SC-FDE schemes are particularly suitable for the uplink transmission (i.e., the transmission from the mobile terminal to the base station), since the envelope fluctuations of the transmitted signals are much lower than those of OFDM schemes [3], [4]. Although, SC-FDE schemes have acceptable performance with a simple linear FDE, the performance is substantially increased with more powerful iterative FDE schemes [5]-[7]. One of the most promising iterative FDE is the IB-DFE (Iterative Block Decision Feedback Equalizer) [8], [9], which can be regarded as a DFE where the feedforward and feedback filters are implemented in the frequency domain. The main problem with QPSK (Quaternary Phase Shift Keying) schemes is that we still need a linear amplifier for SC modulations, since the transmitted signal still has envelope fluctuations ${ }^{1}$.

For very-low-cost mobile terminals, it is desirable to have grossly nonlinear power amplifiers, which are simpler to implement and have higher amplification efficiency and output power. However, these amplifiers are only recommendable when the signal at its input has an almost constant envelope. In this case, OQPSK (Offset QPSK) modulations, also denoted by staggered modulations [10], are strongly recommendable since the transmitted signals can have very low envelope fluctuations or even quasi-constant envelope. It should be pointed out that for QPSK/OQPSK constellations the main degradation due to a nonlinear amplifier is associated to the spectral widening effects (i.e., increased out-of-band radiation levels); due to the reduced dynamic range of OQPSK signals (without zeros crossings) the spectral widening effects are much smaller than with corresponding QPSK signals, even when we employ signals that do not have constant envelope (although we can have similar PAPR (Peak-to-Average Power Ratio) for QPSK and OQPSK signals with high spectral efficiency, such as with a small roll-off square-root raisedcosine filtering).

\footnotetext{
${ }^{1}$ The exception is the case where rectangular pulses are employed, which is usually not employed due to their poor spectral characteristics.
} 
The most famous OQPSK modulation is MSK (Minimum Shift Keying) [11], but GMSK signals (Gaussian MSK) [12] and other CPM signals (Continuous Phase Modulation) [13] can also be written as the sum of OQPSK components [14]. Moreover, this description in terms of OQPSK components can be employed to define TCM-OQAM schemes (Trellis Coded Modulation-Offset Quadrature Amplitude Modulation) with good code gains [15], [16]. Naturally, OQAM signals have significant envelope fluctuations, making them prone to nonlinear distortion effects. However, they can be decomposed as the sum of several OQPSK signals that can be separately amplified almost without distortion [17]. Nevertheless, we should be cautions when extending to offset modulations receivers that were designed for non-offset modulations [10], [18].

Combining OQPSK modulations with SC-FDE schemes seems a natural choice for the uplink of highly power efficient broadband wireless systems. Since OQPSK signals with constant or quasi-constant envelope have bandwidth much higher than the minimum Nyquist band, the FDE should be designed with several samples per symbol to take full advantage of diversity effects, inherent to the larger transmission bandwidth and/or to be able to cope with synchronization errors [19].

This paper considers frequency-domain receiver design for OQPSK and OQAM schemes. It is shown that the FDE designed for non-offset modulations are not suitable for offset modulations due to the residual IQI (In-phase/Quadrature Interference), i.e. the interference between in-phase (I) and quadrature $(\mathrm{Q})$ components at the sampling instants. Although the time-domain receivers especially designed for offset modulations such as those of [10], [18] can address this problem, their complexity is too high for the severely time-dispersive channels we are considering. Therefore, we propose several FDE designs where there is no IQI at the sampling instants and iterative FDE receivers with IQI cancellation.

This paper is organized as follows: section II, characterizes linear and iterative FDE designs for non-offset and offset modulations, as well as the multiplicity concept. Several methods to mitigate IQI in linear FDE are proposed in section III. In section IV is presented an alternative approach to minimize the overall interference. The characterization, relevant properties and performance results of iterative FDE with IQI cancellation, are presented in section V. Finally, the paper is summarized in section VII.

\section{LINEAR AND ITERATIVE FDE DESIGNS WITH OVERSAMPLING}

\section{A. FDE for QPSK Schemes}

The length- $N$ data block to be transmitted is $\left\{a_{n} ; n=\right.$ $0,1, \ldots, N-1\}$, where $a_{n}=a_{n}^{I}+j a_{n}^{Q}$ is the $n$th data symbol with $a_{n}^{I}= \pm 1$ and $a_{n}^{Q}= \pm 1$ associated to the "in-phase" and "quadrature" bits, respectively. For a QPSK scheme the transmitted signal is

$$
x_{B P}(t)=\operatorname{Re}\left\{x(t) \exp \left(j 2 \pi f_{c} t\right)\right\},
$$

where $f_{c}$ is the carrier frequency and $x(t)$ is its the complex envelope, given by

$$
x(t)=\sum_{n=-N_{C P}}^{N-1} a_{n} r\left(t-n T_{s}\right),
$$

with $r(t)$ denoting the adopted pulse shape and $N_{C P}$ the length of the cyclic prefix required for an efficient FDE implementation [4] (it is assumed that the block $\left\{a_{n} ; n=\right.$ $0,1, \ldots, N-1\}$ is periodic with period $N$, i.e., $\left.a_{n}=a_{n+N}\right)$.

Let us first assume that $x(t)$ is the complex envelope of a QPSK scheme with pulse shape $r(t)$ and data symbols $a_{n}, n=0,1, \ldots, N-1$. If a given block is sampled with rate $J / T_{s}$, i.e., with $J$ samples per symbol, then the samples associated to the useful part of the block (i.e., without cyclic prefix) are $\left\{\check{x}_{n} ; n=0,1, \ldots, J N-1\right\}$, with $\check{x}_{n} \triangleq x\left(n T_{s} / J\right)$ (it is assumed that the rate $J / T_{s}$ is large enough to avoid aliasing effects). Since $x(t)$ is cyclostationary [20] (i.e., $E[x(t) x(t-\tau)]$ is periodic in $t$, with period $T_{s}$ ), the frequency-domain block associated to $\left\{\check{x}_{n} ; n=0,1, \ldots, J N-1\right\}$ is $\left\{\check{X}_{k} ; k=\right.$ $0,1, \ldots, J N-1\}=$ DFT $\left\{\check{x}_{n} ; n=0,1, \ldots, J N-1\right\}$, with

$$
\check{X}_{k}=\check{A}_{k} \check{R}_{k}
$$

where $\left\{\check{R}_{k} ; k=0,1, \ldots, J N-1\right\}$ is the DFT of $\left\{\check{r}_{n} \triangleq\right.$ $r((n-J N / 2) T / J) ; n=0,1, \ldots, J N-1\}$ (without loss of generality, we assume that $r(t)$ is centered in 0 and $\left\{\check{A}_{k} ; k=\right.$ $0,1, \ldots, J N-1\}$ is the DFT of $\left\{\check{a}_{n} ; n=0,1, \ldots, J N-1\right\}$, with

$$
\check{a}_{n}= \begin{cases}a_{n^{\prime}}, & n=J n^{\prime} \\ 0, & \text { otherwise }\end{cases}
$$

It can easily be shown that

$$
\check{A}_{k}=\frac{1}{J} A_{k},
$$

where $\left\{A_{k} ; k=0,1, \ldots, N-1\right\}$ denotes the DFT of $\left\{a_{n} ; n=\right.$ $0,1, \ldots, N-1\}$ (once again the time-domain and frequencydomain blocks associated to the data are periodic with period $N)$. This means that there is an implicit multiplicity in the frequency-domain block when the adopted pulse shape has bandwidth above the Nyquist band (i.e., when $\check{R}_{k}$ is not restricted to $N$ non-zero samples), since the frequency-domain sample $A_{k}$ is repeated in several $\check{X}_{k}$ samples (which are separated by multiples of $N$ ) as shown in Figure 1. This multiplicity can be regarded as a frequency diversity effect that can be used by the FDE.

The received block is sampled with the rate $J / T_{s}$, the cyclic prefix is removed and the resulting blocks, $\left\{\check{y}_{n} ; n=\right.$ $0,1, \ldots, J N-1\}$, are passed to the frequency domain, leading to the blocks $\left\{\check{Y}_{k} ; k=0,1, \ldots, J N-1\right\}$. If the cyclic prefix is longer than the overall channel impulse response length then

$$
\check{Y}_{k}=\check{A}_{k} \check{H}_{k}+\check{N}_{k},
$$

where $\check{H}_{k}$ is the overall channel impulse response for the $k$ th subcarrier (which includes the adopted pulse shape, the channel and the $1 / J$ factor inherent to (5)) and $\check{N}_{k}$ is the corresponding noise component.

For a linear FDE the output is

$$
\tilde{A}_{k}=\sum_{q=0}^{J-1} \check{F}_{k+q N} \check{Y}_{k+q N}, \quad k=0,1, \ldots, N-1
$$




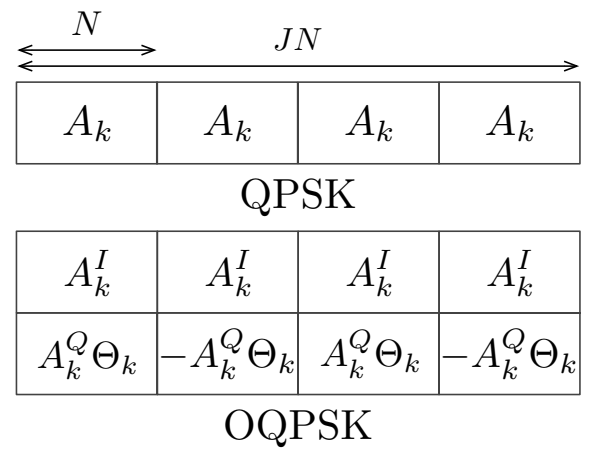

Fig. 1. Frequency-domain multiplicity in QPSK (up) and OQPSK (down) signals.

where the FDE coefficients, optimized under the MMSE criterion (Minimum Mean Squared Error), are given by

$$
\begin{aligned}
\check{F}_{k}=\frac{\check{H}_{k}^{*}}{\alpha+\sum_{q=0}^{J-1}\left|\check{H}_{(k \bmod N)+q N}\right|^{2}}, & \\
k & =0,1, \ldots, J N-1,
\end{aligned}
$$

with $(\cdot)^{*}$ denoting complex conjugate, $x$ mod $y$ denoting the modulo operator, i.e. the remainder of $x / y$, and

$$
\alpha=E\left[\left|\check{N}_{k}\right|^{2}\right] / E\left[\left|\check{A}_{k}\right|^{2}\right]
$$

denoting the inverse of the SNR (Signal-to-Noise Ratio).

For a given iteration of the IB-DFE, the FDE output is

$$
\tilde{A}_{k}=\sum_{q=0}^{J-1} \check{F}_{k+q N} \check{Y}_{k+q N}-B_{k} \bar{A}_{k}, \quad k=0,1, \ldots, N-1,
$$

where $\left\{\bar{A}_{k} ; k=0,1, \ldots, N-1\right\}$ is the DFT of $\left\{\bar{a}_{n} ; n=\right.$ $0,1, \ldots, N-1\}$, with $\bar{a}_{n}$ denoting the average values of $\tilde{a}_{n}$ associated to the previous iteration, conditioned to the FDE output. For QPSK constellations with Grey mapping these average values can be obtained as described in [21] and for 16QAM constellations with Grey mapping they can be obtained as described in [22]. For general $M^{2}$-QAM constellations we can employ the approach of [23] where the constellation symbols are expressed as a function of the corresponding bits as follows:

$$
a_{n}=\sum_{m=1}^{\varphi} 2^{\varphi-m} \prod_{q=1}^{m} b_{n}^{(q) I}+j \sum_{m=1}^{\varphi} 2^{\varphi-m} \prod_{q=1}^{m} b_{n}^{(q) Q},
$$

with $M=2^{\varphi}, b_{n}^{(q) I}= \pm 1$ and $b_{n}^{(q) Q}= \pm 1$. The log-likelihood ratio of the $m$ th bit of the $n$th transmitted symbol is given by

$$
L L R_{n}^{(m)}=\log \left(\frac{\sum_{a \in \Phi_{1}^{(m)}} \exp \left(-\frac{\left|\widetilde{a}_{n}-a\right|^{2}}{2 \sigma^{2}}\right)}{\sum_{a \in \Phi_{0}^{(m)}} \exp \left(-\frac{\left|\widetilde{a}_{n}-a\right|^{2}}{2 \sigma^{2}}\right)}\right),
$$

where $\Phi_{1}^{(m)}$ and $\Phi_{0}^{(m)}$ are the constellation's subsets associated to the symbols with the $m$ th bit at 1 or 0 , respectively and

$$
\sigma^{2}=\frac{1}{2 N} \sum_{n=0}^{N-1} E\left[\left|\widetilde{a}_{n}-a_{n}\right|^{2}\right]
$$

To obtain the average symbol values conditioned to the FDE output, $\bar{a}_{n}$, we need the average bit values conditioned to the FDE output, $\bar{b}_{n}^{(m)}$. They can be related as

$$
\bar{b}_{n}^{(m)}=\tanh \left(\frac{L L R_{n}^{(m)}}{2}\right)=\rho_{n}^{(m)} \hat{b}_{n}^{(m)},
$$

with $\hat{b}_{n}^{(m)}$ denoting the estimate of $b_{n}^{(m)}$ and $\rho_{n}^{(m)}$ its reliability. From (11), (14) and assuming uncorrelated bits due to the usage of a suitable interleaver, we have

$$
\bar{a}_{n}=\sum_{m=1}^{\varphi} 2^{\varphi-m} \prod_{q=1}^{m} \bar{b}_{n}^{(q) I}+j \sum_{m=1}^{\varphi} 2^{\varphi-m} \prod_{q=1}^{m} \bar{b}_{n}^{(q) Q} .
$$

Having in mind the results of [24] it can be shown that the IB-DFE coefficients are given by

$$
\begin{aligned}
\check{F}_{k}=\frac{\kappa \check{H}_{k}^{*}}{\alpha+\left(1-\rho^{2}\right) \sum_{q=0}^{J-1}\left|\check{H}_{(k \bmod N)+q N}\right|^{2}}, \\
\quad k=0,1, \ldots, J N-1,
\end{aligned}
$$

and

$$
B_{k}=\sum_{q=0}^{J-1} \check{F}_{k+q N} \check{H}_{k+q N}-1, \quad k=0,1, \ldots, N-1,
$$

respectively, where $\kappa$ is selected to ensure that

$$
\frac{1}{N} \sum_{k=0}^{J N-1} \check{F}_{k} \check{H}_{k}=1
$$

$\alpha$ is defined by (9), and the correlation coefficient $\rho$ that regards block-wise reliability of the decisions used in the feedback loop is given by

$$
\rho=\frac{E\left[\bar{a}_{n} a_{n}^{*}\right]}{E\left[\left|a_{n}\right|^{2}\right]} \approx \frac{E\left[\bar{a}_{n} \widehat{a}_{n}^{*}\right]}{E\left[\left|\widehat{a}_{n}\right|^{2}\right]} .
$$

For general $M^{2}$-QAM constellations we have

$$
\rho=\frac{\sum_{m=1}^{\varphi} 4^{\varphi-m}\left(\prod_{q=1}^{m} \rho_{n}^{(q) I} \prod_{q=1}^{m} \rho_{n}^{(q) Q}\right)}{2 \sum_{m=1}^{\varphi} 4^{\varphi-m}} .
$$

\section{B. Multiplicity in OQPSK Signals}

For an OQPSK scheme we still have

$$
x_{B P}(t)=\operatorname{Re}\left\{x(t) \exp \left(j 2 \pi f_{c} t\right)\right\},
$$

but with the complex envelope given by

$$
\begin{aligned}
x_{p}(t)=\sum_{n=-N_{C P}}^{N-1} a_{n}^{I} r\left(t-n T_{s}\right) & \\
& +j \sum_{n=-N_{C P}}^{N-1} a_{n}^{Q} r\left(t-n T_{s}-T_{d}\right),
\end{aligned}
$$

Clearly, the OQPSK signal can be regarded as the sum of two binary PAM (Pulse Amplitude Modulation) signals each with symbols (bits) separated by $T_{s}$ (the average bit rate is $1 / 2 T_{s}$ ), and with an offset $T_{d}$ between them (which usually is $T_{s} / 2$ ).

Although, the in-phase and quadrature components of OQPSK signals are cyclostationary with period $T_{s}$, the 